\title{
Can one detect passage of small black hole through the Earth?
}

\author{
I. B. Khriplovich, ${ }^{1, *}$ A. A. Pomeransky, ${ }^{1, t}$ N. Produit, ${ }^{2, \text { t }}$ and G. Yu. Ruban ${ }^{1,5}$ \\ ${ }^{1}$ Budker Institute of Nuclear Physics, 630090, Novosibirsk, Russia, and Novosibirsk University \\ ${ }^{2}$ INTEGRAL Science Data Center, 16, Chemin d'Ecogia, CH-1290 Versoix, Switzerland
}

The energy losses of a small black hole passing through the Earth are examined. In particular, we investigate the excitations in the frequency range accessible to modern acoustic detectors. The main contribution to the effect is given by the coherent sound radiation of the Cherenkov type.

\section{INTRODUCTION}

Primordial black holes could arise at the early stages of the Universe evolution when the matter density was very high. But which of them could survive since those times? The problem is that too light primordial black holes have already evaporated due to their thermal radiation. Well-known estimates [1] demonstrate that the masses of the survivors should exceed $10^{15} \mathrm{~g}$.

As a step to study the possibility to detect the passage of so small black hole through the Earth (or some other planet, or the Moon), we analyze here the effects arising during such a passage with a velocity comparable to that of the planet.

It turns out that for a supersonic black hole, i.e. for that with velocity exceeding the speed of sound, the main effect is a coherent excitation of sound waves in the matter, the acoustic analogue of Cherenkov radiation. These waves can be in principle observed by seismic measurements.

\section{COHERENT SOUND GENERATION BY A SUPERSONIC BLACK HOLE}

We start with the dynamics of mechanical deformations and excitation of sound waves caused by the passage of a primordial black hole through matter. The deformation can be conveniently described by the displacement vector $\mathbf{u}$ with respect to the positions of the matter points in the absence of black hole. For small deformations $\mathbf{u}$ satisfies the linear wave equation:

$$
\ddot{\mathbf{u}}-c_{s}^{2} \Delta \mathbf{u}=\mathbf{g} .
$$

Here $\mathbf{g}$ is the gravitational acceleration created by the black hole, and $c_{s}$ is the speed of sound. We note that only the longitudinal sound modes are excited by the gravitational force since $\mathbf{g}$ is the gradient of a scalar potential: $\mathbf{g}=\nabla \phi$.

The gravitational acceleration $\mathbf{g}$ satisfies the Gauss law:

$$
\nabla \mathbf{g}=-4 \pi G[M \delta(\mathbf{r}-\mathbf{v} t)+\delta \rho]
$$

Here $M$ and $\mathbf{v}$ are the black hole mass and velocity, respectively; the deviation $\delta \rho$ of matter density from its equilibrium value in the absence of a black hole is related to the displacement vector as follows:

$$
\delta \rho=-\rho \nabla \mathbf{u}
$$

The divergence of Eq. (1) is

$$
\ddot{\psi}-c_{s}^{2} \Delta \psi-4 \pi G \rho \psi=-4 \pi G M \delta(\mathbf{r}-\mathbf{v} t),
$$

where $\psi=\nabla \mathbf{u}$. The corresponding equation for the Fourier transforms $\psi_{\mathbf{k}}$ reads

$$
\ddot{\psi}_{\mathbf{k}}+\varepsilon \dot{\psi}_{\mathbf{k}}+c_{s}^{2} k^{2} \psi_{\mathbf{k}}-4 \pi \rho G \psi_{\mathbf{k}}=-4 \pi M G \exp (-i \mathbf{k} \mathbf{v} t)
$$

\footnotetext{
*Electronic address: khriplovich@inp.nsk.su

†Electronic address: a.a.pomeransky@inp.nsk.su

${ }^{\ddagger}$ Electronic address: Nicolas.Produit@obs.unige.ch

$\S$ Electronic address: gennady.ru@gmail.com
} 
we have introduced here an infinitesimal damping $\varepsilon$ which corresponds to the retarded solution and is equivalent to a small viscosity term in the wave equation. The solution of Eq. (4) is

$$
\psi_{\mathbf{k}}=\frac{4 \pi G M \exp (-i \omega t)}{\omega^{2}+i \omega \varepsilon-c_{s}^{2} k^{2}+4 \pi G \rho}
$$

with $\omega=\mathbf{k v}=k_{\|} v$.

The decelerating force applied to the black hole is equal to

$$
M \mathbf{g}=-4 \pi i \rho M G \int \mathbf{k} \psi_{\mathbf{k}} \exp (i \omega t) \frac{d^{3} \mathbf{k}}{(2 \pi)^{3} k^{2}} .
$$

The radiation intensity, or the rate of energy loss by the black hole due to its elastic interaction with matter, is

$$
\begin{aligned}
I_{e l}=-M \mathbf{g} \mathbf{v} & =(4 \pi G M)^{2} \rho i \int \frac{\omega}{\omega^{2}+i \omega \varepsilon-c_{s}^{2} k^{2}+4 \pi G \rho} \frac{d^{3} \mathbf{k}}{(2 \pi)^{3} k^{2}} \\
& =(4 \pi G M)^{2} \rho i \int \frac{\omega}{\omega^{2}+i \omega \varepsilon-c_{s}^{2} k^{2}+4 \pi G \rho} \frac{d \omega d^{2} \mathbf{k}_{\perp}}{(2 \pi)^{3} v k^{2}} .
\end{aligned}
$$

Now we use well-known relation

$$
\frac{1}{x+i \varepsilon}=P \frac{1}{x}-i \pi \delta(x)
$$

where $P$ is the principal value of an integral. Here the contribution of the principal value is an odd function of $\omega$ and therefore vanishes after integration over $\omega$. Thus, we obtain

$$
I_{e l}=2(G M)^{2} \rho \int \frac{d^{2} \mathbf{k}_{\perp}}{v k^{2}} \int_{-\infty}^{\infty} \delta\left(\omega^{2}\left(1-c_{s}^{2} / v^{2}\right)-c_{s}^{2} k_{\perp}^{2}+4 \pi G \rho\right) \omega d \omega .
$$

In our case the black hole moves faster than sound, $v>c_{s}$, and the $\delta$-function gives a non-vanishing contribution only if $k_{\perp}>\omega_{p} / c_{s}$, where $\omega_{p}^{2}=4 \pi G \rho$. Then,

$$
I_{e l}=\frac{2 \pi(G M)^{2} \rho}{v\left(1-c_{s}^{2} / v^{2}\right)} \int_{\left(\omega_{p} / c_{s}\right)^{2}}^{k_{1}^{2}} \frac{d k_{\perp}^{2}}{k^{2}}=2 \pi(G M)^{2} \rho / v \int_{\left(\omega_{p} / c_{s}\right)^{2}}^{k_{1}^{2}} \frac{d k_{\perp}^{2}}{k_{\perp}^{2}-\omega_{p}^{2} / v^{2}}=2 \pi(G M)^{2} \rho / v \int_{\left(\omega_{p} / c_{s}\right)^{2}}^{k_{1}^{2}} \frac{d k_{\perp}^{2}}{k_{\perp}^{2}} ;
$$

we recall here that $v \gg c_{s}$. This integral diverges logarithmically at large momentum transfers from the black hole to the matter, when the scattering gets inelastic. Fortunately, if we are interested in the total energy losses, elastic plus inelastic (see below), the exact value $k_{1}^{2}$ of such critical momentum transfers is inessential.

\section{INELASTIC SCATTERING. TOTAL MECHANICAL LOSSES}

We start here with the opposite limiting case, that of large momentum transfers, when the matter can be considered as a collection of free particles (of mass $m$, number density $n$, and mass density $\rho=m n$ ). The differential cross-section for scattering of a black hole on such a particle is

$$
\frac{d \sigma}{d \Omega}=\frac{1}{4}\left(\frac{G M}{v^{2}}\right)^{2} \frac{1}{\sin ^{4} \theta / 2}
$$

The corresponding decelerating force looks formally as

$$
F=n m v^{2} \frac{1}{4}\left(\frac{G M}{v^{2}}\right)^{2} 2 \pi \int_{0}^{\pi} \frac{d \theta \sin \theta(1-\cos \theta)}{\sin ^{4} \theta / 2}=2 \pi \rho \frac{(G M)^{2}}{v^{2}} \int_{0}^{\pi} d \theta \frac{\cos \theta / 2}{\sin \theta / 2} .
$$

In fact, the typical scattering angles here are small, so that

$$
\int_{0}^{\pi} d \theta \frac{\cos \theta / 2}{\sin \theta / 2}=\ln \frac{\theta_{\max }^{2}}{\theta_{\min }^{2}}=\ln \frac{k_{\perp \max }^{2}}{k_{\perp \min }^{2}}
$$


Obviously, $k_{\perp \max } \simeq 1 / a$, where $a$ is the typical interatomic distance in the matter. As to the minimum momentum transfer $k_{\perp \text { min }}$ at which one can neglect the binding of the matter constituents, it coincides as obviously, at least in the order of magnitude, with the maximum momentum transfer $k_{1}^{2}$ at which the interaction of a black hole with matter remains elastic (see equation (9) ). Thus, the rate of inelastic energy loss by a black hole is

$$
I_{\text {inel }}=F v=2 \pi(G M)^{2} \rho / v \ln \frac{1}{k_{1}^{2} a^{2}}
$$

And finally, the total rate of energy loss is

$$
I_{t o t}=I_{e l}+I_{\text {inel }}=4 \pi(G M)^{2} \rho / v \ln \frac{c_{s}}{\omega_{p} a} .
$$

With the accepted logarithmic accuracy, this total rate is independent of the critical momentum transfer $k_{1}$. On the other hand, for any reasonable choice of $k_{1}$, the elastic energy loss dominates strongly, $I_{e l} \gg I_{i n e l}$. It is worth mentioning also that the logarithm in (13) is really large, about 35. An expression for $I_{t o t}$ close to (13), but with a different logarithmic factor, was obtained previously by K. Penanen [2].

To estimate the energy $\Delta E$ released by a black hole passing through the Earth, this rate should be multiplied by the time of the passage, $\tau=L / v$. For numerical estimates we assume that the equilibrium density of matter is $\rho=6 \mathrm{~g} / \mathrm{cm}^{3}$, the path $L$ is about the Earth diameter, $L \sim 10^{4} \mathrm{~km}$, and the velocity of black hole is $v \sim 30 \mathrm{~km} / \mathrm{s}$. At last, for a black hole with mass $M \sim 10^{15} \mathrm{~g}$ this energy loss constitutes about

$$
\Delta E \sim 4 \times 10^{9} \mathrm{~J}
$$

Let us note that this energy is much smaller than that released at the explosion of a 10 kiloton atomic bomb

$$
\Delta E_{\text {bomb }} \sim 5 \times 10^{13} \mathrm{~J} .
$$

Besides, when comparing the energy released by a black hole (not only (14), but also some other contributions to it considered below) with the energy of an atomic bomb, one should keep in mind that the source of $\Delta E_{b o m b}$ is practically point-like, while $\Delta E$ is spread along a path $L \sim 10^{4} \mathrm{~km}$.

\section{CONVERSION OF BLACK HOLE RADIATION INTO SOUND WAVES}

One more source of the energy transfer from a light black hole to the matter (though not its kinetic energy discussed above, but the internal one) is the black hole radiation. Of course, for our purpose we have to consider the emission of $\gamma$ and $e^{ \pm}$only (but not gravitons and neutrinos). Using the results of [3], we obtain under the same assumptions $\left(M \sim 10^{15} \mathrm{~g}, L \sim 10^{4} \mathrm{~km}\right.$, and $\left.v \sim 30 \mathrm{~km} / \mathrm{s}\right)$ the following estimate for the total radiation loss of such black hole:

$$
\Delta E_{\text {rad }} \sim 1.5 \times 10^{12} \mathrm{~J}
$$

One of the possible mechanisms for the conversion of radiation into sound waves, which permits of rather reliable theoretical analysis, is as follows. The radiation absorbed by matter increases the temperature along the path of the source. This results in the inhomogeneous and non-stationary thermal expansion of the matter and thus in the emission of acoustic waves. The matter is treated as a liquid (the case of a solid medium could be considered analogously), and the well-known relations

$$
\dot{\rho}+\operatorname{div} \rho \mathbf{v}=0, \quad \rho \dot{\mathbf{v}}=-\nabla p
$$

result in the following equation

$$
\ddot{\rho}-\Delta p=0
$$

for density $\rho$ and pressure $p$. The variations of density, pressure, and temperature are related as follows:

$$
\delta \rho=\left(\frac{\partial \rho}{\partial p}\right)_{T} \delta p+\left(\frac{\partial \rho}{\partial T}\right)_{p} \delta T=\frac{1}{c_{s}^{2}} \delta p-\rho \beta \delta T ;
$$

here $\beta=-1 / \rho(\partial \rho / \partial T)_{p}$ is the coefficient of thermal expansion. This allows one to eliminate $\rho$ from (18):

$$
\frac{1}{c_{s}^{2}} \ddot{p}-\Delta p-\rho \beta \ddot{T}=0 .
$$


Neglecting the thermal conductivity, we rewrite $\rho \dot{T}$ as $W / C$, where $W$ is the power density and $C$ is the specific heat. Thus, such effect due to heating is described by the following equation for pressure (derived previously in Ref. [4]):

$$
\frac{1}{c_{s}^{2}} \ddot{p}-\Delta p=\frac{\beta}{C} \dot{W}
$$

A black hole can be treated as a point-like source of radiation with intensity $I$, so that in our case $W=I \delta(\mathbf{r}-\mathbf{v} t)$.

Let us consider now the mechanical energy of the matter:

$$
E_{m}=\int\left(\frac{p^{2}}{2 \rho c_{s}^{2}}+\frac{\rho v^{2}}{2}\right) d V .
$$

The intensity of the sound radiation coincides with the rate of energy loss:

$$
\frac{d E_{m}}{d t}=\int\left(\frac{p \dot{p}}{\rho c_{s}^{2}}+\rho \mathbf{v} \dot{\mathbf{v}}\right) d V=\int\left(\frac{p \dot{p}}{\rho c_{s}^{2}}-\mathbf{v} \nabla p\right) d V .
$$

We use here the Euler equation $\rho \dot{\mathbf{v}}=-\nabla p$ to rewrite the last term. Then, integrating by parts and using the continuity equation we arrive at:

$$
\frac{d E_{m}}{d t}=\int \frac{p}{\rho}\left(\frac{\dot{p}}{c_{s}^{2}}-\dot{\rho}\right) d V=\int \frac{\beta p W}{\rho C} d V .
$$

With a point-like source $W=I \delta(\mathbf{r}-\mathbf{v} t)$, the integration is performed easily:

$$
\frac{d E_{m}}{d t}=\frac{\beta I}{\rho C} p(\mathbf{v} t)
$$

where $\mathrm{p}(\mathbf{v t})$ is the pressure at the point of the black hole location. Now the Fourier transformed Eq. (21) is

$$
p_{\omega, \mathbf{k}}=\frac{\beta I}{C}(-i \omega) \frac{2 \pi \delta(\omega-\mathbf{k v})}{k^{2}-(\omega+i \varepsilon)^{2} / c_{s}^{2}} .
$$

Here we have introduced again an infinitesimal damping $\varepsilon$. With $\varepsilon \rightarrow 0$, the frequency Fourier component of the pressure at the black hole location is

$$
\begin{gathered}
p_{\omega}=\int \frac{d \mathbf{k}}{(2 \pi)^{3}} p_{\omega, \mathbf{k}}=\frac{\beta I}{C} \int \frac{d \mathbf{k}}{(2 \pi)^{2}} \frac{-i \omega \delta(\omega-\mathbf{k} \mathbf{v})}{k^{2}-(\omega+i \varepsilon)^{2} / c_{s}^{2}} \longrightarrow \frac{\beta I \omega}{C} \int \frac{d \mathbf{k}}{(2 \pi)^{2}} \pi \delta(\omega-\mathbf{k v}) \delta\left(k^{2}-\omega^{2} / c_{s}^{2}\right) \\
=\frac{\beta I \omega}{4 v C} \int_{0}^{\infty} d k_{\perp}^{2} \delta\left(k_{\perp}^{2}+\omega^{2} / v^{2}-\omega^{2} / c_{s}^{2}\right)=\frac{\beta I \omega}{4 v C} .
\end{gathered}
$$

The intensity of the sound radiation at given frequency $\omega$ is related to $p_{\omega}$ as follows:

$$
\frac{d E_{m}}{d t d \omega} d \omega=2 \frac{\beta I}{\rho C} p_{\omega} \frac{d \omega}{2 \pi}
$$

the overall factor 2 in this expression corresponds to the fact that frequencies of both signs, $\omega$ and $-\omega$, are taken here into account. Finally, we arrive at the following result for the spectral intensity of the sound waves:

$$
\frac{d E_{m}}{d t d \omega}=\left(\frac{\beta I}{C}\right)^{2} \frac{\omega}{4 \pi v \rho} .
$$

The total energy radiated in this way at the frequency $\omega$, during the passage of a black hole through the Earth, can be conveniently written as

$$
\frac{d E_{m}}{d \omega}=\left(\frac{\beta \Delta E_{r a d}}{C}\right)^{2} \frac{\omega}{4 \pi L \rho}
$$

we go over in this expression from the intensity $I$ of the black hole radiation to the total energy $\Delta E_{\text {rad }}$ emitted during the passage through the Earth: $\Delta E_{\text {rad }}=I L / v$.

Curiously, this sound radiation occurs only if $v>c_{s}$, as it is obvious from the last line of Eq. (27). In other words, this is also a sort of Cherenkov effect. 


\section{MATTER EXCITATIONS AND SENSITIVITY OF SEISMIC DETECTORS}

To detect a mini-black hole passing through the Earth, one has to study seismic vibrations induced by this passage. The sensitivity of appropriate seismic detectors is confined to the frequencies in the interval around $\omega_{\min } \sim 1 \mathrm{~Hz}$ and $\omega_{\max } \sim 100 \mathrm{~Hz}$.

To determine the frequency distribution of the acoustic Cherenkov radiation, we come back to formula (8) and perform at first integration over $\mathbf{k}_{\perp}$. With $v \gg c_{s}$ and $\omega \gg \omega_{p}$, the result is

$$
d I_{e l}=4 \pi(G M)^{2}(\rho / v) \frac{d \omega}{\omega} .
$$

Thus, the energy of the vibrations excited in the frequency interval $\omega_{\min } \div \omega_{\max }$ is

$$
\Delta E^{\omega}=4 \pi(G M)^{2} L \rho / v^{2} \ln \frac{\omega_{\max }}{\omega_{\min }} .
$$

For the discussed frequency interval, $1 \div 100 \mathrm{~Hz}$, it constitutes numerically

$$
\Delta E^{\omega} \sim 5 \times 10^{8} \mathrm{~J}
$$

or about $1 / 10$ of the total energy (14).

As to the seismic waves generated by the black hole radiation, their total energy for frequencies $\omega<\omega_{\max }$ is, according to (30),

$$
E_{m}^{\omega}=\left(\frac{\beta \Delta E_{r a d}}{C}\right)^{2} \frac{\omega_{\max }^{2}}{8 \pi L \rho}
$$

With $C=1 \mathrm{~J} \mathrm{~g}^{-1} \mathrm{~K}^{-1}$ and $\beta=0.5 \cdot 10^{-4} \mathrm{~K}^{-1}$, we obtain $E_{m}^{\omega} \sim 40 \mathrm{~J}$. So, this effect is much less than that of the Cherenkov sound radiation (33).

There is in fact one more mechanism by which the energy of black hole radiation is transformed into the mechanical energy of matter. It is as follows. Of course, in the rest frame of a black hole its radiation is isotropic, so that the total momentum of radiated particles is equal to zero. However, it is not so in the rest frame of the Earth. In it the momentum carried away by particles radiated by a black hole with velocity $v$ per unit time can be estimated as $I v / c^{2}$ (here $c$ is the speed of light). All this momentum is absorbed by the matter together with the radiation itself. Therefore, the effective force of interaction between the black hole and matter is

$$
F \sim \frac{I v}{c^{2}} \sim \frac{\Delta E_{r a d} v^{2}}{c^{2} L}
$$

Thus, the total energy transferred due to this pressure from the black hole to matter along the path $L$ can be estimated as

$$
\Delta E_{p r} \sim F L \sim \Delta E_{\text {rad }} \frac{v^{2}}{c^{2}} \sim 10^{-8} \Delta E_{\text {rad }} \sim 10^{4} \mathrm{~J} .
$$

However, the portion of energy released in this way in the frequency region $\omega<\omega_{\max } \sim 100 \mathrm{~Hz}$ is much less. Its crude estimate looks as follows. The typical absorption length $r_{0}$ for $\gamma$ and $e^{ \pm}$is in our case about $3 \mathrm{~cm}$. Simple estimates demonstrate that the relative concentration of the defects in matter created by this radiation is small everywhere. (In this respect as well, our problem of the seismic waves, generated by the black hole radiation, differs from that for the underground explosion of an atomic bomb: in the last case the region of complete destruction of the matter is measured at least by meters.) Thus, in the present case $r_{0}$ is the only length scale at our disposal. This region of radius $r_{0}$ propagates in the matter with the velocity $v$ of a black hole. Then the typical frequency of thus created perturbations in the matter can be estimated as

$$
\omega_{0} \sim v / r_{0} \sim 10^{6} \mathrm{~Hz}
$$

The frequencies $\omega$, we are interested in, are much smaller, $\omega_{\max } \ll \omega_{0}$. It looks natural to assume that in our low frequency ("infrared") region the radiation intensity is governed by the phase space considerations. With the essentially two-dimensional propagation of the perturbation around the line of flight of a black hole, the phase space can be considered also as a two-dimensional one. Then one can assume that the frequency spectrum in the lowfrequency region of interest looks as $\omega^{2} / \omega_{0}^{2}$, and the "useful" portion of black hole radiation, which directly induces seismic vibrations in the frequency interval $1 \div 100 \mathrm{~Hz}$, can be estimated as

$$
\Delta E_{p r}^{\omega} \sim E_{r a d} \frac{v^{2}}{c^{2}} \frac{\omega_{\max }^{2}}{\omega_{0}^{2}}
$$


which is negligibly small. Anyway, even the total energy (36) transferred in this way is less than the useful part (33) of the sound Cherenkov radiation.

One more effect we wish to mention is as follows. It was pointed out long ago [5] that, due to the positron annihilation and the capture of Compton- and $\delta$-electrons by a cosmic-ray electron-photon shower, an excess of electrons arises in this shower. Due to the excess, a shower gets effectively charged, creates correspondingly a macroscopic electromagnetic field, and radiates coherently.

Obviously, analogous effects take place in principle during the passage of a radiating black hole through the Earth (of course, the intensities of electrons and positrons emitted by a black hole itself are equal). Let us estimate the field created in this way by a black hole. We assume that the excess of electrons created here per second is about the same as the total production rate of particles by a black hole $\dot{N}$. Then the magnetic field at the distance on the order of the absorption length $r_{0}$ from the black hole can be estimated as

$$
B \sim e \frac{v}{c} \frac{\dot{N}}{c r_{0}} .
$$

It falls down as $1 / r^{2}$ with the distance from the black hole. With $\dot{N} \sim 10^{21} \mathrm{~s}^{-1}, v \sim 30 \mathrm{~km} / \mathrm{s}$, and $r_{0} \sim 3 \mathrm{~cm}$, this magnetic field is about $0.3 \mathrm{Gs}$ only. The possibility to use such a signal for detecting the passage of a light black hole through the Earth does not look realistic.

To summarize, the seismic signal of the passage of a light black hole through the Earth in the frequency interval $1 \div 100 \mathrm{~Hz}$ is strongly dominated by the sound Cherenkov radiation, and its total energy in this interval can be estimated as

$$
\Delta E^{\omega} \sim 5 \times 10^{8} \mathrm{~J} .
$$

We wish to point out in conclusion that though the effects of radiation damage contribute negligibly into the seismic signal, they can create quite a distinct pattern in crystalline material. The dose deposited is estimated as

$$
\frac{\Delta E_{\mathrm{rad}}}{\rho L r_{0}^{2}} \sim 10^{5} \text { Gy } \quad(1 \mathrm{~Gy}(\text { Gray })=1 \mathrm{~J} / \mathrm{kg}) .
$$

It creates a long tube of heavily radiative damaged material, which should stay recognizable for geological time.

[1] S.W. Hawking, Commun. Math. Phys. 43, 199 (1975).

[2] K. Penanen, private communication.

[3] D.N. Page, Phys. Rev. D 13, 198 (1976).

[4] P.G. Westervelt, R.S. Larson, J. Acous. Soc. Amer. 54, 121 (1973).

[5] G.A. Askaryan, Zh. Eksp. Teor. Phys. 41, 616 (1961) [Sov. Phys. JETP 41, (1961)]. 